\title{
AN EXPERIMENTAL PLATFORM FOR STATISTICAL FAULT DIAGNOSIS IN PROPELLED MECHANICAL SYSTEMS: AN ACADEMIC EXERCISE
}

\author{
Leonardo Acho \\ Department of Mathematics \\ Universitat Politècnica de Catalunya \\ -BarcelonaTech (ESEIAAT), \\ Terrasa, Spain. \\ Spain \\ leonardo.acho@upc.edu
}

\author{
Pablo Buenestado \\ Department of Mathematics \\ Escola d'Enginyeria de Barcelona Est, \\ Universitat Politcnica \\ de Catalunya- \\ BarcelonaTech \\ Spain \\ pablo.buenestado@upc.edu
}

\author{
Article history: \\ Received 16.01.2019, Accepted 10.06.2019
}

\begin{abstract}
Statistical data analysis for fault diagnosis in mechanical systems is a fundamental tool, for instance, in applied mechanical engineering. In order to capture a feasible data set, a well designed electronic instrumentation and excitation system signal stages are mandatory. Hence, one objective of this paper is to develop a lowcost vibration sensor based on an inductive LC-tank oscillator (a resonant inductive-capacitive electronic circuit carefully designed to produce an harmonic electrical signal), and then to tune an effective excitation system signal to our experimental platform. This platform uses a propelled drone motor mounted on a beam structure to emulate a propelled rotating machine. Essentially, two data set were acquired. One for the healthy behaviour of the developed system, and the other for a programmed faulty scenario. This defective case was realized by introducing a small mechanical fault in one blade extreme of the mechanical propelled system. To note, this faulty scenario is almost impossible to de-
\end{abstract}

duce by just seen the raw data. The other objective of this paper is to analyze the obtained data sets by utilizing a statistical data analysis tool. Then, by employing box-plot diagrams, the healthy and faulty cases become evidenced. Finally, and due to we are proposing a low-cost academic experimental platform for fault diagnosis based on data analysis, our platform's toll was around 120 euros. Hence, this platform results applicable to teach data analysis from dynamical systems.

\section{Key words}

Propelled rotating machines, statistics, fault diagnosis, electronic instrumentation, signal processing.

\section{Introduction}

Propelled rotating machines are widely employed in many engineering applications such as wind turbines [Luo et al., 2014], drones [Floreano and Wood, 2015; Belyavskyi et al., 2017], ship drivers [Bosschers et al., 2008], rotating tools [Glowacz, 2018], and so on. On the other hand, fault diagnosis applied to this kind of mechanical devices has been extensively 
studied. For instance, a fault diagnosis based on data analysis has been proposed in [Vidal et al., 2015] by invoking a controller compensation design. However, this fault scheme is just oriented to detect faults on the pitch-blade actuator device, as other techniques do [Ruiz et al., 2018; Vidal et al., 2014; Badihi and Zhang, 2018; Chen et al., 2013a; Tutivén et al., 2018]. In [Orchard and Vachtsevanos, 2007] describes the implementation of an online particle-filtering-based system for fault diagnosis in a turbine engine. In [Hernandez-M. et al., 2017] analyzes the application of a time-frequency technique for detection of an unbalance fault in a Wind turbine, etc..

Nevertheless, in propelled rotating machines, the most common on field-operating system fault is due to a damage on the propeller (or blade) body, see for instance [Shajiee et al., 2014; Oberholster and Heyns, 2006; Abdelrhman et al., 2013; Abdelrhman et al., 2012; Zeng et al., 2019]. Even when there exit many statistical methods on fault diagnosis based on data analysis, such as deep learning [Helbing and Ritter, 2018], Kalman filtering theory [Cho et al., 2018], bi-stable stochastic resonance theory [He et al., 2019], fuzzy logic and intelligent systems [Kuo, 1995], etcetera, we can still invoke fundamental statistical tools, like box-plot diagrams, for fault diagnosis to propeller rotating machines. Therefore, one main objective of this paper is to implement a low-cost experimental platform on propelled rotating machines to acquire feasible data sets for fault diagnosis. Hence, the principal challengers involve to instrument a economical vibration sensor, and to tune an appropriate system excitation signal. Our designed vibration sensor is based on an LC-tank oscillator where its output amplitude-frequency depends on the proximity variation of a magnet carefully located on the support structure of our propelled mechanism. Then, by using a (modified) peak-detector circuit (actually, this circuit has been previously used in [Pujol V. and Acho Z., 2014] to obtain a usable vibration sensor), a vibration signal is received. Additionally, the cost of this experimental platform is completely accessible to any engineering student. On the other hand, to manipulate the actuated propeller, realized by using a DC-Motor, an offset triangular excitation signal is programmed by utilizing a function generator. This excitation signal induces a kind of structure vibration and depending on the propeller dynamic.

On the other side, the development of low cost experimental platforms for engineering applications has also been of academic interest. For instance, in [Jiang et al., 2006], a low-cost hybrid experimental platform applied to test digital power controllers has been evolved, including nonlinear systems analysis [Acho, 2013; Acho, 2014]. In [Ponce de L. P. et al., 2018], an affordable experimental platform to essay MPPT algorithms to photo-voltaic panels has been granted. And in [Ren et al., 2008], an experimental platform to teach digital electronic concepts has been proposed. Hence, the main preference of this paper is to grant an low-priced experimental platform too.

The rest of this document is structured as follows. Section 2 describes our experimental platform, its material and methods whereas Section 3 reports our experimental results. Finally, Sections 4 and 5 state the discussion and conclusions of our main contributions, respectively.

\section{Platform Description: Material and Methods}

This section goes on a general description of our implemented experimental platform including its material and methods. At this point, Figure 1 presents the main parts of a fault diagnosis assemble based on data analysis [Norton and Karczub, 2003], and Figure 2 displays a picture of our apparatus. The propelled system was extracted from a drone toy. Therefore, the platform main parts are: 1) the propelled mechanism, 2) the designed vibration sensor, and 3) the function generator (FG) device.

Additionally, Figure 3 gives the electronic circuit of the (inductive) LC-tank oscillator (a resonant inductivecapacitive electronic circuit carefully designed to automatic generate an harmonic electrical signal) realized as our vibration sensor. Here, the movement of the attached permanent magnet on the system structure support manipulates the amplitude-frequency of this circuit's response. Then, by utilizing a (modified) peak-detector scheme (the diode-resistance-capacitor 
configuration), the vibration-amplitude information is extracted and available at the $V$ sen point. Here, the principal components are the inductive elements. For this, we employ a choke-coil taken out from an old TV monitor. Figure 4 shows its picture. This choke-coil has two inductive elements mounted on a $H$-ferrite core structure. Actually, this idea to develop a position sensor based on inductive elements was initially motivated by [Kej1k et al., 2004; Passeraub et al., 1997].

On the other face, the electronic circuit to drive the propelled system via a DC-Motor is drawn in Figure 5. This circuit is the basic inverted-summer-amplifier where its out is power amplified by using a power NPN-transistor on its emitter-follower configuration. Moreover, in our electronic executions, we use the $L M 741$ operational-amplifiers and supplied by a \pm 12 volts power source. Finally, Figures 6 and 7 exhibit the pictures of the two scenarios cases for the blade structure employed in our experiments: 1) the healthy, and the 2) faulty blade profiles.

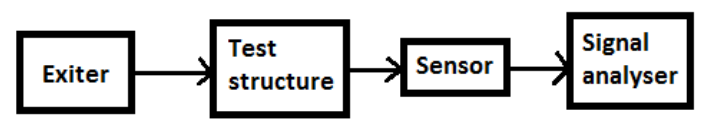

Figure 1. Main parts of a structure fault diagnosis setup. From the academic point of view, this system structure is important to conceive to realize future developments on fault system diagnosis.

\section{Experimental Results}

This section analyzes the obtained experimental results from our developed platform. As an example, Figure 8 displays the measured experimental outcomes related to the faulty stage. Figures 9 and 10 display the histograms and probability density approximation functions of the vibration sensor data related to each scenario, the healthy and the faulty ones. Additionally, Figure 11 displays the corresponding box-plots, where

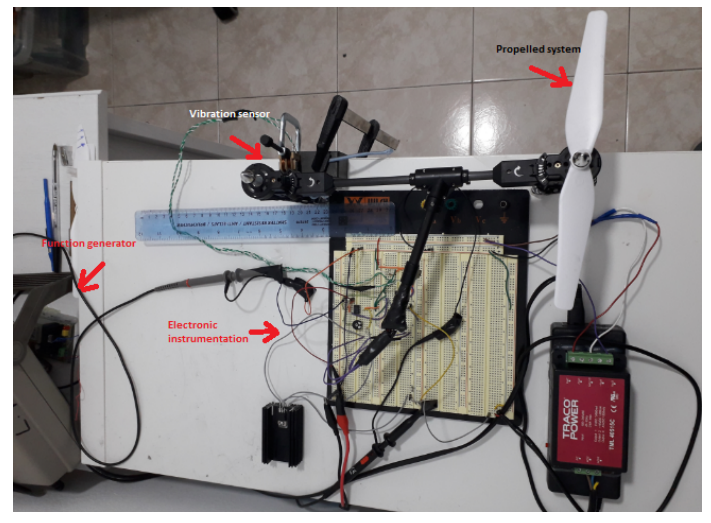

Figure 2. The proposed experimental platform.

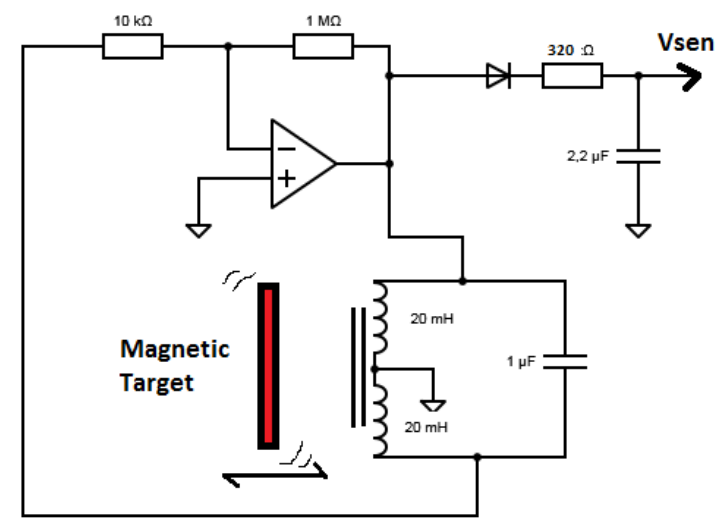

Figure 3. The electronic circuit of our vibration sensor based on an LC-tank oscillator. The peak-detector system is composed by the diode-resistance-capacitor configuration. The employed diode is the $1 N 4001$ one.

it is clearly evidenced that the feasible obtained data can distinguish each scenario. Finally, the outcome data signals were acquired by using the Picoscope2000-series digital oscilloscope and then analyzed by utilizing the open-source $R$ software. 


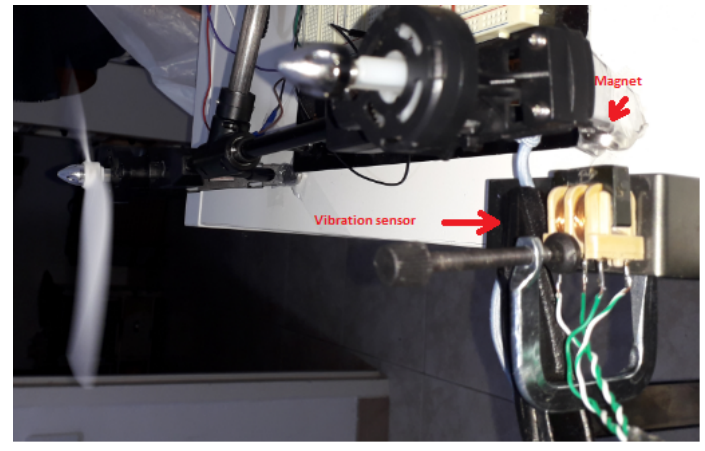

Figure 4. A picture of the implemented vibration sensor. The choke-coil device is here appreciated.

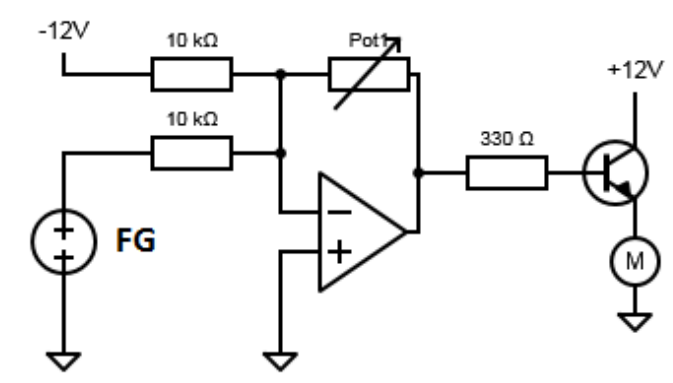

Figure 5. The electronic circuit to drive the propelled system via a DC-Motor (M). The potentiometer Pot1 is adjusted to supply a maximum voltage value to the DC-motor of around 8 volts. The NPN-transistor is a power one (MJE3055T). FG is the signal generated by the generator function instrument.

\section{Discussion}

From the engineering implementation point of view, the electronic realization to acquire feasible data for fault diagnosis in mechanical systems is a key stage design along with an appropriate excitation system command (see, for instance, [Haridas and Pawar, 2018; Chen et al., 2013b]). Our proposed platform involves these important parts by incorporating a vibration sensor based on an LC-tank oscillator (hence, electronic engineering students may find it attractive as an application of it), and by programming an appropriate reference trajectory used by the propelled system's actuator via the DC-Motor. In our approach, we use

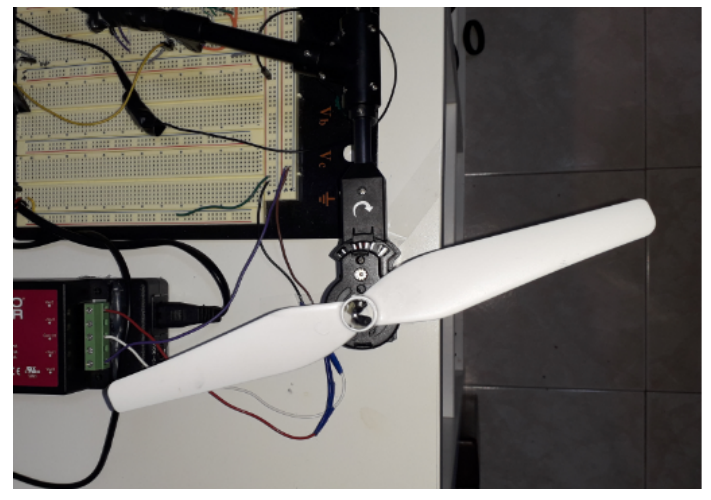

Figure 6. The evoked healthy propeller.

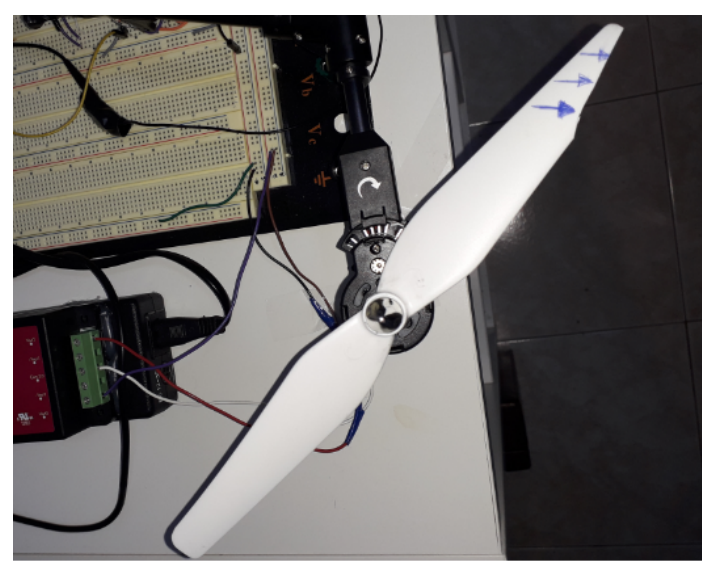

Figure 7. The appealed faulty propeller.

a triangular reference excitation signal to drive the propelled mechanism. In this way, the angular velocity of the propelled system's driver was periodically varied from a slow to a kind of fast rotation angular velocity of the blade system. Intuitively speaking, this may induce an enough rich structural vibration frequency content on the system's support. See a video on it at https://youtu.be/Mv5tcbUIKzs .

Moreover, Figure 12 shows our vibration sensor performance when the cited magnet is manually moved to the choke-coil head and then backward and repeated several times between a distance of about one centimeter. To note, this sensor operates whatever is the polarity of the magnet seen by the sensor. Finally, 


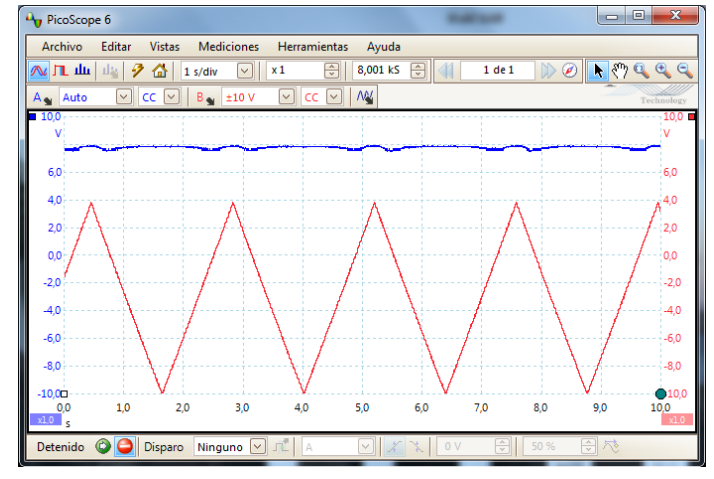

Figure 8. Measured signals for the faulty case. In blue is the signal from our vibration sensor and in red is the generated signal by the function generator.

Data Histogram: Healthy case

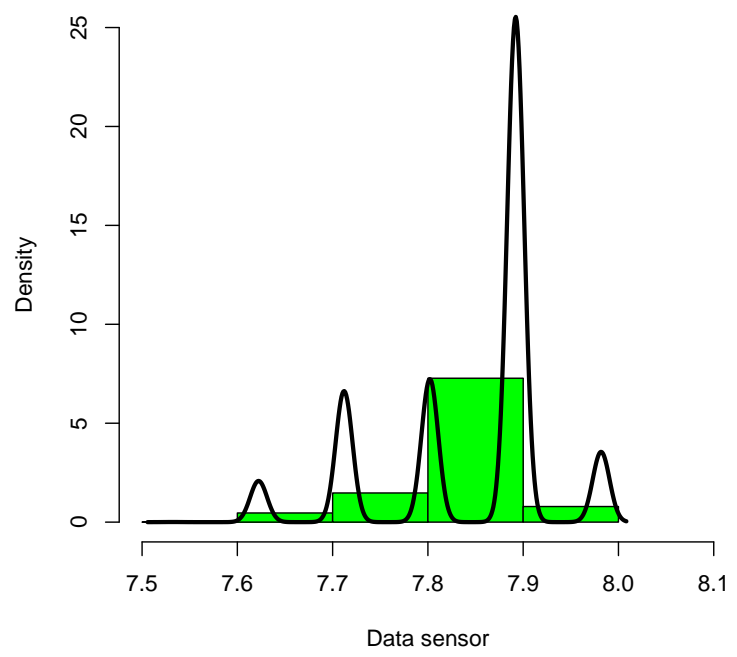

Figure 9. Data histogram: the healthy case.

as a future research direction is to develop an automatic system for fault diagnosis to our experimental platform.

\section{Conclusions}

In this paper a low-cost experimental platform was proposed for fault diagnosis in propelled rotating
Data Histogram: Faulty case

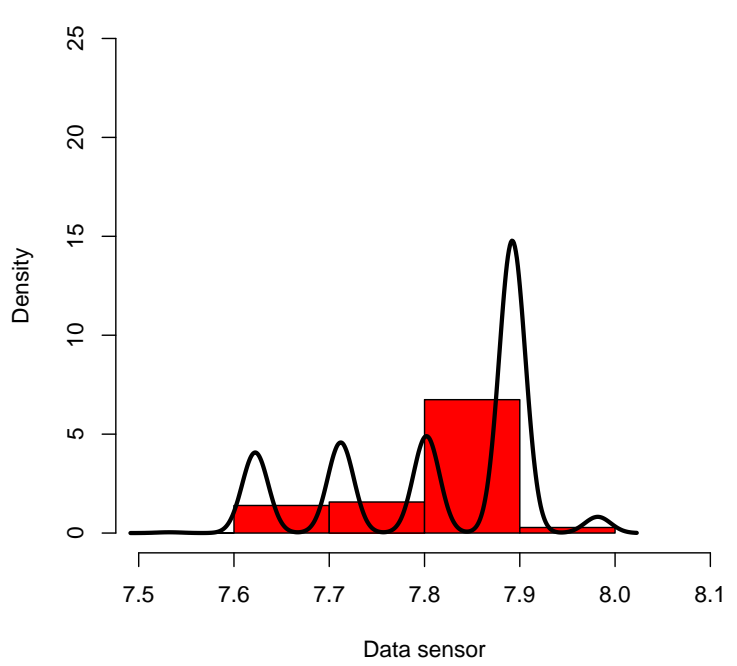

Figure 10. Data histogram: the healthy scenario.

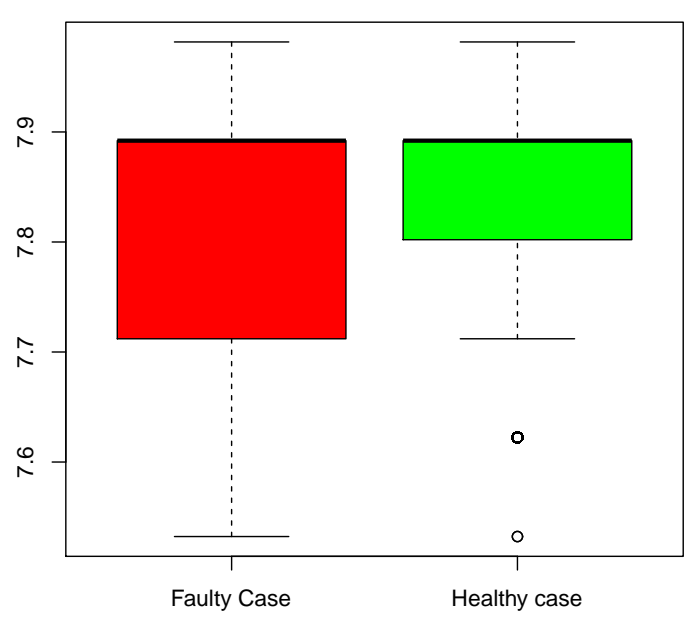

Figure 11. Box-plot diagrams.

machines. We think that by using this platform, engineering students can practice on acquiring and 


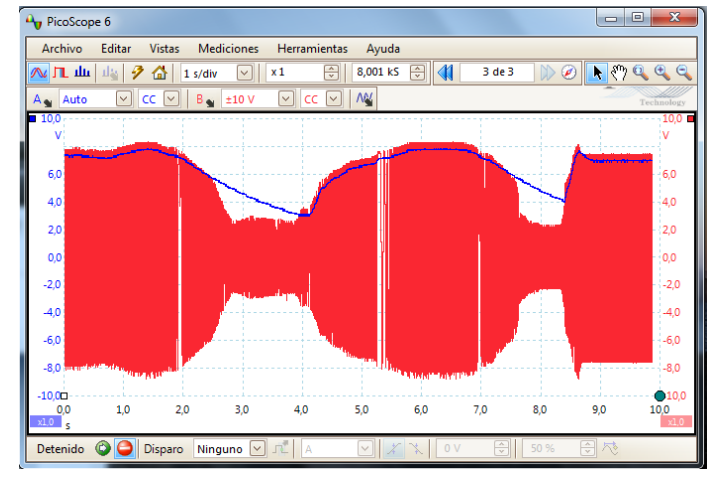

Figure 12. Vibration sensor performance. In red is the oscillator's output and in blue is the response given by the peak-detector circuit.

analyzing data for fault diagnosis in rotating machines based on statistical methods. Additionally, in our experiments, we evidence the potential of box-plot diagrams for fault diagnosis in mechanical systems. Finally, as future developments include to realize a velocity control to the propelled system and to incorporate state-of-the-art on data processing diagnosis such as machine learning, etc.

\section{Funding}

This research was partially funded by the Spanish Ministry of Economy and Competitiveness/Fondos Europeos de Desarrollo Regional (MINECO/FEDER) with grant number DPI2015-64170-R.

\section{References}

Abdelrhman, A. M., Leong, M. S., Hee, L. M., and Ngui, W. K. (2013). Application of wavelet analysis in blade faults diagnosis for multi-stages rotor system. In Applied Mechanics and Materials, volume 393, pages 959-964. Trans Tech Publ.

Abdelrhman, A. M., Leong, M. S., Saeed, S. A. M., Al Obiadi, S. M., et al. (2012). A review of vibration monitoring as a diagnostic tool for turbine blade faults. In Applied Mechanics and Materials, volume 229, pages 1459-1463. Trans Tech Publ.

Acho, L. (2013). Modeling and synchronization of multivibrator oscillators. Cybernetics and Physics, 2(1):5-9.
Acho, L. (2014). A delta modulator technique to drive the chaotic duffing oscillator. Cybernetics and Physics, 3(1):4-8.

Badihi, H. and Zhang, Y. (2018). Fault-tolerant individual pitch control of a wind turbine with actuator faults. IFAC-PapersOnLine, 51(24):1133-1140.

Belyavskyi, A. O., Tomashevich, S. I., and Andrievsky, B. (2017). Application of 2dof quadrotor-based laboratory testbed for engineering education. In Proc. 2017 Mediterranean Conference on Control and Automation (MED), pages 939-944. IEEE.

Bosschers, J., Vaz, G., Starke, A., and van Wijngaarden, E. (2008). Computational analysis of propeller sheet cavitation and propeller-ship interaction. In Proc. 2008 RINA conference (MARINE CFD), Southampton, UK, pages 26-27.

Chen, B., Matthews, P. C., and Tavner, P. J. (2013a). Wind turbine pitch faults prognosis using a-priori knowledge-based anfis. Expert Systems with Applications, 40(17):6863-6876.

Chen, Z., Yang, Y., Xie, Y., Guo, B., and Hu, Z. (2013b). Non-contact crack detection of high-speed blades based on principal component analysis and euclidian angles using optical-fiber sensors. Sensors and Actuators A: Physical, 201:66-72.

Cho, S., Gao, Z., and Moan, T. (2018). Model-based fault detection, fault isolation and fault-tolerant control of a blade pitch system in floating wind turbines. Renewable Energy, 120:306-321.

Floreano, D. and Wood, R. J. (2015). Science, technology and the future of small autonomous drones. Nature, 521(7553):460.

Glowacz, A. (2018). Recognition of acoustic signals of commutator motors. Applied Sciences, 8(12):2630.

Haridas, P. D. and Pawar, P. M. (2018). Experimental analysis of thin walled composite rotor blade model from structural health monitoring aspect. Materials Today: Proceedings, 5(2):5679-5691.

He, C., Li, H., Li, Z., and Zhao, X. (2019). An improved bistable stochastic resonance and its application on weak fault characteristic identification of centrifugal compressor blades. Journal of Sound and Vibration, 442:677-697.

Helbing, G. and Ritter, M. (2018). Deep learning for fault detection in wind turbines. Renewable and Sustainable Energy Reviews, 98:189-198. 
Hernandez-M., S. Z., Hernandez-O., U., AmezquitaSanchez, J. P., Valtierra-R., M., and Granados-L., D. (2017). Detection of unbalance in a wind turbine by using wavelet packet transform and vibration signals. In 2017 IEEE International Autumn Meeting on Power, Electronics and Computing (ROPEC), pages 1-6. IEEE.

Jiang, Z., Dougal, R. A., Leonard, R., Figueroa, H., and Monti, A. (2006). Hardware-in-the-loop testing of digital power controllers. In Proc. 2006 Twenty-First Annual IEEE on Applied Power Electronics Conference and Exposition (APEC), pages 6-pp. IEEE.

Kej1k, P., Kluser, C., Bischofberger, R., and Popovic, R. S. (2004). A low-cost inductive proximity sensor for industrial applications. Sensors and Actuators A: Physical, 110(1-3):93-97.

Kuo, R. (1995). Intelligent diagnosis for turbine blade faults using artificial neural networks and fuzzy logic. Engineering Applications of Artificial Intelligence, 8(1):25-34.

Luo, N., Vidal, Y., and Acho, L. (2014). Wind turbine control and monitoring. Springer.

Norton, M. P. and Karczub, D. G. (2003). Fundamentals of noise and vibration analysis for engineers. Cambridge university press.

Oberholster, A. and Heyns, P. S. (2006). On-line fan blade damage detection using neural networks. Mechanical systems and signal processing, 20(1):78-93.

Orchard, M. E. and Vachtsevanos, G. J. (2007). A particle filtering-based framework for real-time fault diagnosis and failure prognosis in a turbine engine. In Proc. 2007 Mediterranean Conference on Control \& Automation (MED), pages 1-6. IEEE.

Passeraub, P. A., Rey-M., G., Besse, P., Lorenz, H., and Popovic, R. (1997). Inductive proximity sensor with a flat coil and a new differential relaxation oscillator. Sensors and Actuators A: Physical, 60(1-3):122-126.

Ponce de L. P., N., Acho, L., and Rodellar, J. (2018). Design and experimental implementation of a hysteresis algorithm to optimize the maximum power point extracted from a photovoltaic system. Energies, 11(7):1866.

Pujol V., G. and Acho Z., L. (2014). Semi-active control of flexible structures using acceleration information via peak-detector systems. In Proc. 2014 6th Conference on Structural Control and Monitoring (6WCSCM), pages 1893-1901. Centre Internacional de Mètodes Numèrics en Enginyeria (CIMNE).

Ren, L., Guo, Z., Jiang, Y., and Wu, H. (2008). Research and practice of experimental simulation platform for course of digital logic. In Proc. 2008 The 9th International Conference for Young Computer Scientists, pages 2541-2545. IEEE.

Ruiz, M., Mujica, L. E., Alférez, S., Acho, L., Tutivén, C., Vidal, Y., Rodellar, J., and Pozo, F. (2018). Wind turbine fault detection and classification by means of image texture analysis. Mechanical Systems and Signal Processing, 107:149-167.

Shajiee, S., Pao, L. Y., and McLeod, R. R. (2014). Monitoring ice accumulation and active de-icing control of wind turbine blades. In Wind Turbine Control and Monitoring, pages 193-230. Springer.

Tutivén, C., Vidal, Y., Acho, L., and Rodellar, J. (2018). Fault detection and isolation of pitch actuator faults in a floating wind turbine. IFAC-PapersOnLine, 51(24):480-487.

Vidal, Y., Acho, L., Luo, N., and Tutiven, C. (2014). Hardware in the loop wind turbine simulator for control system testing. In Wind Turbine Control and Monitoring, pages 449-466. Springer.

Vidal, Y., Tutivén, C., Rodellar, J., and Acho, L. (2015). Fault diagnosis and fault-tolerant control of wind turbines via a discrete time controller with a disturbance compensator. Energies, 8(5):4300-4316.

Zeng, J., Chen, K., Ma, H., Duan, T., and Wen, B. (2019). Vibration response analysis of a cracked rotating compressor blade during run-up process. $M e$ chanical Systems and Signal Processing, 118:568583. 